\title{
A Retrospective Database Study Comparing Diabetes-Related Medication Adherence and Health Outcomes for Mail-Order Versus Community Pharmacy
}

\author{
Phil Schwab, PhD; Patrick Racsa, MS; Karen Rascati, RPh, PhD; Marc Mourer, MBA; \\ Yunus Meah, PharmD; and Karen Worley, PhD
}

\begin{abstract}
BACKGROUND: Adherence to oral antihyperglycemic agents (AHAs) is important for managing blood glucose levels and avoiding hospitalizations or diabetes complications. Previous studies have found that use of mailorder pharmacy dispensing channels results in greater adherence than use of community pharmacies, but the link between use of mail-order pharmacies and improved clinical outcomes has not been established.
\end{abstract}

OBJECTIVE: To compare the effect of mail-order and community pharmacy use on adherence to oral AHAs, hemoglobin A1c (A1c) level, and glycemic control, as well as emergency department (ED) and inpatient hospital use.

METHODS: This retrospective cohort study of administrative claims data from January 1, 2008, to December 31, 2016, included patients with Medicare Advantage Prescription Drug plan coverage with $\geq 2$ claims for the same oral AHA and a diagnosis of type 2 diabetes mellitus (T2DM). Patients were indexed to the start of the most advanced oral AHA identified to begin study observations at the start of a new treatment and assigned to mail-order or community pharmacy cohorts based on which channel dispensed $\geq 80 \%$ of their oral AHA claims; all others were excluded. Mail-order and community pharmacy patients were 1:1 propensity score matched. Matched cohorts were compared on proportion of days covered (PDC), adherence (PDC $\geq 0.8$ ), A1c level, glycemic control, and ED and inpatient use for measurement periods of $12,24,36$, and 48 months post-index.

RESULTS: 19,307 mail-order and 19,307 community pharmacy users were matched. PDC was higher for mail-order pharmacy users at 12 months ( 0.93 vs. $0.82, P<0.001)$ and sustainable through 48 months ( 0.87 vs. 0.77 , $P<0.001)$. Adherence was also greater for mail-order pharmacy patients through 12 months ( $86 \%$ vs. $68 \%, P<0.001)$ and sustainable through 48 months ( $78 \%$ vs. $62 \%, P<0.001)$. Glycemic control as $A 1 \mathrm{c}<7 \%$ was not significantly different, but control as A1c $<8 \%$ was greater for mail-order pharmacy users at 12 months ( $91 \%$ vs. $89 \%, P=0.006)$ and was greater through 36 months ( $93 \%$ vs. $89 \%, P=0.043$ ). Effects on A1c level were not evident. Mail-order pharmacy users were less likely to have an ED visit within 12 months ( $26 \%$ vs. $28 \%, P<0.0001)$, and the difference was observed through 36 months ( $50 \%$ vs. $54 \%, P<0.0001)$. Similarly, fewer mail-order pharmacy users had an inpatient hospitalization within 12 months ( $17 \%$ vs. $19 \%, P<0.0001)$, and the difference was observed through 48 months ( $43 \%$ vs. $47 \%, P=0.009$ ).

CONCLUSIONS: The results of the study demonstrate a benefit to patients who use mail-order pharmacies for chronic medications to treat T2DM. The study identified greater glycemic control, lower ED use, and lower hospitalization among individuals using mail-order pharmacies. These positive outcomes were evident in the near term and sustained over time.

J Manag Care Spec Pharm. 2019;25(3):332-40

Copyright $\odot 2019$, Academy of Managed Care Pharmacy. All rights reserved.

\section{What is already known about this subject}

Mail-order pharmacy users achieve greater adherence than do community pharmacy users.

Previous research among patients with type 2 diabetes supports the link between mail-order pharmacy use and greater adherence.

\section{What this study adds}

Mail-order and community pharmacy users may be inherently different, so this study used novel methods to control for potential differences and to focus on the effects of interest.

Adherence and other study outcomes were measured over a longer period of time than previous research comparing mail-order and community pharmacy users

Consistent with previous studies, mail-order pharmacy users were found to have greater adherence to oral antihyperglycemic agents, but this study also observed the link between mail-order pharmacy use and adherence over a longer span of time.

A dherence to chronic, oral antihyperglycemic agents (AHAs) for treating type 2 diabetes mellitus (T2DM) is important for managing blood glucose levels and avoiding hospitalizations or diabetes complications. ${ }^{1-3}$ Conveniences such as 90-day supply refills are known to improve on-hand drug supply, so home delivery of medications and more frequent use of 90-day supply fills by mail-order pharmacies relative to community pharmacies should lead to greater adherence, resulting in improved glycemic control and fewer avoidable health care events. ${ }^{3-5}$

Previous studies have established a link between mail-order pharmacy use and greater adherence. A systematic review cited 14 of 15 studies that observed greater adherence among mail-order pharmacy users relative to community pharmacy users. ${ }^{6}$ The evidence is also consistent within T2DM, specifically, where published studies show that mail-order pharmacy users achieve greater adherence to AHAs over approximately 12 months based on data from administrative pharmacy claims. $^{7-12}$ The study by Schmittdiel et al. (2013) went a step further by incorporating medical claims with pharmacy claims to evaluate outcomes for patients with T2DM. ${ }^{5}$ The study found 


\section{A Retrospective Database Study Comparing Diabetes-Related Medication Adherence and Health Outcomes for Mail-Order Versus Community Pharmacy}

that fewer mail-order pharmacy patients (aged $<65$ years) had an emergency department (ED) visit compared with community pharmacy users, but the effect was not detected among patients aged $\geq 65$ years. ${ }^{5}$ No differences were detected between mail-order and community pharmacy users on the proportion with an inpatient hospitalization, regardless of patient age. ${ }^{5}$

The present study adds to the current body of evidence regarding the influence of mail-order pharmacy use and makes several important contributions. First, the study evaluated adherence, glycemic control, ED visits, and inpatient hospitalizations. The study observed these measures over 12 months, similar to previous studies, but also included assessments spanning up to 4 years. Additionally, the study used novel measures and methods to focus on the effects of interest while minimizing baseline differences between mail-order and community pharmacy users.

\section{Methods}

\section{Data Source}

The study sample was selected from the Humana Research Database. The database has national coverage with a high proportion of individuals from Texas, Florida, and Ohio and is one of the largest Medicare Advantage Prescription Drug (MAPD) plan claims databases in the United States. For this study, only the MAPD population was examined. Stand-alone prescription drug plan and commercial populations within the database were not included. Claims data included information regarding physician, outpatient, and ED visits and hospitalizations. Pharmacy claims data included detailed information for each prescription fill. Hemoglobin Alc (Alc) measurements were also included for the subset of patients with laboratory results available. The full study period was from January 1, 2008, through December 31, 2016.

\section{Study Design and Patient Selection}

This retrospective, longitudinal cohort study of patients enrolled in MAPD plans identified eligible patients based on 2 or more paid pharmacy claims for the same oral AHA between January 1, 2009, and December 31, 2015, with the first such claim assigned as the index date. Patients were required to have at least 12 months of pre-index and 12 months of post-index continuous health plan enrollment to allow for sufficient observation periods. The sampling methodology identified patients new to AHA therapy entirely or initiating an oral AHA that the individual had not previously used. A method was employed that prioritized new starts, treatment additions, or switches involving newer or second-line medications to maximize the identification of index dates where drug class initiation was evident. The order of identification was such that sodium-glucose cotransporter 2 inhibitor claims were identified first, followed by dipeptyl peptidase-4 inhibitors, thiazolidinediones, sulfonylureas, and metformin. Patients identified based on initiation of meglitinides or alpha-glucosidase inhibitors were excluded owing to extremely low numbers. Because this study focused on oral AHAs, patients were excluded if they used insulin or any other injectable for T2DM (e.g., amylin agonists and glucagon-like peptide-1 receptor agonists) before the index date or within 12 months after the index date.

A pre-index medical claim with a diagnosis for T2DM (International Classification of Diseases, Ninth Revision, Clinical Modification [ICD-9-CM] codes 250.x0 and 250.x2; International Classification of Diseases, Tenth Revision, Clinical Modification [ICD-10-CM] code E11.x) was required. Patients were excluded if there was baseline evidence of type 1 diabetes mellitus via insulin or insulin pump use. Patients were also excluded due to diagnosis for pregnancy or gestational diabetes (ICD-9/10-CM 630.xx-679.xx or v22.x-v24.x; Oxx.x), polycystic ovarian syndrome (ICD-9/10-CM 256.4; E28.2), or steroid-induced diabetes (ICD-9/10-CM 251.8, E932.x; E09.x) during the pre-index period. Any patient aged $<19$ or $>89$ years as of the index date was also excluded.

The pre-index baseline period was used to characterize patients for matching purposes and to observe a baseline Alc measurement. The follow-up period was used to measure adherence to oral AHAs, to observe follow-up Alc measurements, and to measure ED and inpatient hospital use. For each patient, all pharmacy claims for oral AHAs were labeled for distribution channel based on the dispensing pharmacy. To establish the cohorts, patients were classified as mail-order pharmacy users if $\geq 80 \%$ of their oral AHA claims in the first 12 months post-index were dispensed by a mail-order facility or as community users if the proportion was $\leq 20 \%$. All other patients were excluded from the study.

The study cohorts were propensity-score matched based on previous use of an oral AHA, presence of Alc results at respective observation periods (see Outcomes Measures section), baseline risk scores (RxRisk- $\mathrm{V}^{13}$ and Diabetes Complications Severity Index $\left.[\mathrm{DCSI}]^{14,15}\right)$, baseline health care use, baseline health care costs, age, sex, race, region of residence, population density of residence, income surrogates (Medicaid eligibility and low-income subsidy eligibility), and segmentation classifications based on patients' chronic conditions and interactions with the health care system. Unmatched patients were excluded from the study. The propensity score method aimed to balance the study cohorts in terms of the probability of being a mail-order pharmacy user based on the set of baseline measures included for the propensity score. A 1:1 matching ratio, without replacement, was employed. The nearest neighbor method of propensity score matching within a caliper of 0.10 of the standard deviation of the estimated logit was used to select the matched samples.

The study protocol was reviewed and approved by Schulman and Associates Institutional Review Board (IRB; now Advarra IRB). 


\section{A Retrospective Database Study Comparing Diabetes-Related Medication Adherence and Health Outcomes for Mail-Order Versus Community Pharmacy}

\section{Variable Definitions}

Age was calculated as of the index date based on date of birth. Sex was categorized as male or female. Race/ethnicity was coded as white, black, or other/unknown. Region was recorded as Northeast, Midwest, South, or West based on U.S. census region assignments and was determined using the subject's resident state as of the index date. Population density was identified as urban (population $\geq 50,000)$, suburban $(10,000$ $49,999)$, rural $(<10,000)$, or unknown. ${ }^{16,17}$ Previous use of an oral AHA was identified as $\geq 1$ pharmacy claim for an oral AHA during the pre-index period. Baseline Alc was identified as the most recent result within 6 months pre-index. Baseline use included all ED, inpatient hospital, and outpatient claims that were measured over the full 12-month pre-index period.

DCSI is a medical claims-based index that describes severity of diabetes complications. ${ }^{14,15}$ DCSI values range from 0 to 13 , with greater values indicating greater diabetes complications severity. ${ }^{15}$ DCSI was determined based on diagnostic data from medical claims in the pre-index period. The DCSI score for the study was modified with methodology from Klabunde et al. (2000). ${ }^{18}$ RxRisk-V is a pharmacy claims-based risk index with values ranging from 0 to 43 in the version used for this study, where a higher score indicates greater comorbidity. ${ }^{13,19-25}$ RxRisk-V was determined based on pharmacy claims in the pre-index period.

The patient segmentation measure was based a proprietary categorization, previously developed by Humana, that uses individual-level data to establish groups of patients that can be described as 1 of the following: self-engaged optimist, simplicity-seeking follower, skeptical control seekers, health service maximizers, overwhelmed and reluctant reactors, healthy self-sustainers, auto-pilot participators, and support-seeking participators. Seven of the 8 groups are further divided into those with and without chronic conditions for 15 categories. Accounting for undefined patients, 16 categories for the patient segmentation variable were used in the study to strengthen propensity-score calculations and matching.

Baseline health care costs included plan paid and patient out-of-pocket costs for pharmacy and medical services during the pre-index period. To mitigate the potential for underestimating costs of services provided under capitated arrangements, costs for these services were imputed as the median value (allowed amount) from noncapitated claims matched by procedure, source of billing, and place of service. Costs for all services were adjusted to 2016 dollars using the medical component of the Consumer Price Index.

\section{Outcomes Measures}

Medication adherence during the follow-up period was evaluated using proportion of days covered (PDC) calculated for any oral AHA, where PDC equaled the number of days with an oral AHA drug on-hand divided by the number of days from the index date through the end of the respective measurement period $(12,24,36$, and 48 months or 365, 731, 1,096, and 1,460 days). The number of days with drug therapy on-hand was calculated using a set of rules to avoid double-counting covered days when prescription fills overlapped, as opposed to summing the days of supply for all prescription fills. Adherence was defined as $\mathrm{PDC} \geq 0.8$.

Alc results were identified by Logical Observation Identifiers Names and Codes \#4548-4, and only values 2\% through 18\% were considered as valid for study observations. Alc results documented as $<2 \%$ or $>18 \%$ were ignored for the study. Twelvemonth Alc results were identified as the most recent result within 10-14 months post-index, and 24-, 36-, and 48-month Alc results were the most recent results within 22-26, 34-38, and 46-50 months, respectively. Change in Alc was calculated as the difference between an Alc result at a given post-index time period and baseline Alc. Alc measures at each time period were also evaluated for goal achievement as Alc $<7 \%$ and the less stringent $\mathrm{Alc}<8 \%$, accordingly. These cut-points were chosen because Alc $<7 \%$ is a well-established goal for attainment of glycemic control, and Alc $<8 \%$ is a less stringent goal that may be more suitable for some patients. ${ }^{26,27}$ Additionally, the Healthcare Effectiveness Data and Information Set (HEDIS), a tool to measure health plan performance, includes a measure of the percentage of all adults aged 18-75 years with diabetes who achieve glycemic control as Alc $<8 \%$ within its comprehensive diabetes care measure. ${ }^{28}$ For HEDIS, the proportion of adults who achieve glycemic control as Alc $<7 \%$ only applies to commercial insurance and Medicaid members, but Medicare patients are not included for the measure. ${ }^{28}$

ED and inpatient hospital use, for any cause, were identified based on place of service for medical claims. ED claims with the same service date as an inpatient hospitalization or adjacent to a hospitalization were not counted as an ED visit because they were considered as part of the hospitalization. Inpatient hospitalization claims where the discharge date and the admit dates were the same date (reflecting a transfer) were considered part of a single hospitalization.

\section{Statistical Methods}

Data management and analyses were conducted using SAS enterprise guide version 7.1 (SAS Institute, Cary, NC). The a priori alpha level for all inferential analyses was set at 0.05, and all statistical tests were 2-tailed. Analyses were completed based on follow-up periods of 12, 24, 36, and 48 months for the respective cohorts of patients with continuous enrollment through the follow-up period and who had not started insulin therapy before the end of the respective follow-up period, because insulin use would complicate oral AHA adherence calculations and confuse interpretations of other outcomes.

The average PDC between mail-order and community pharmacy use was compared using paired t-tests based on 12-, 24-, 


\section{A Retrospective Database Study Comparing Diabetes-Related Medication Adherence and Health Outcomes for Mail-Order Versus Community Pharmacy}

\section{FIGURE 1 Study Sample Identification and Attrition}

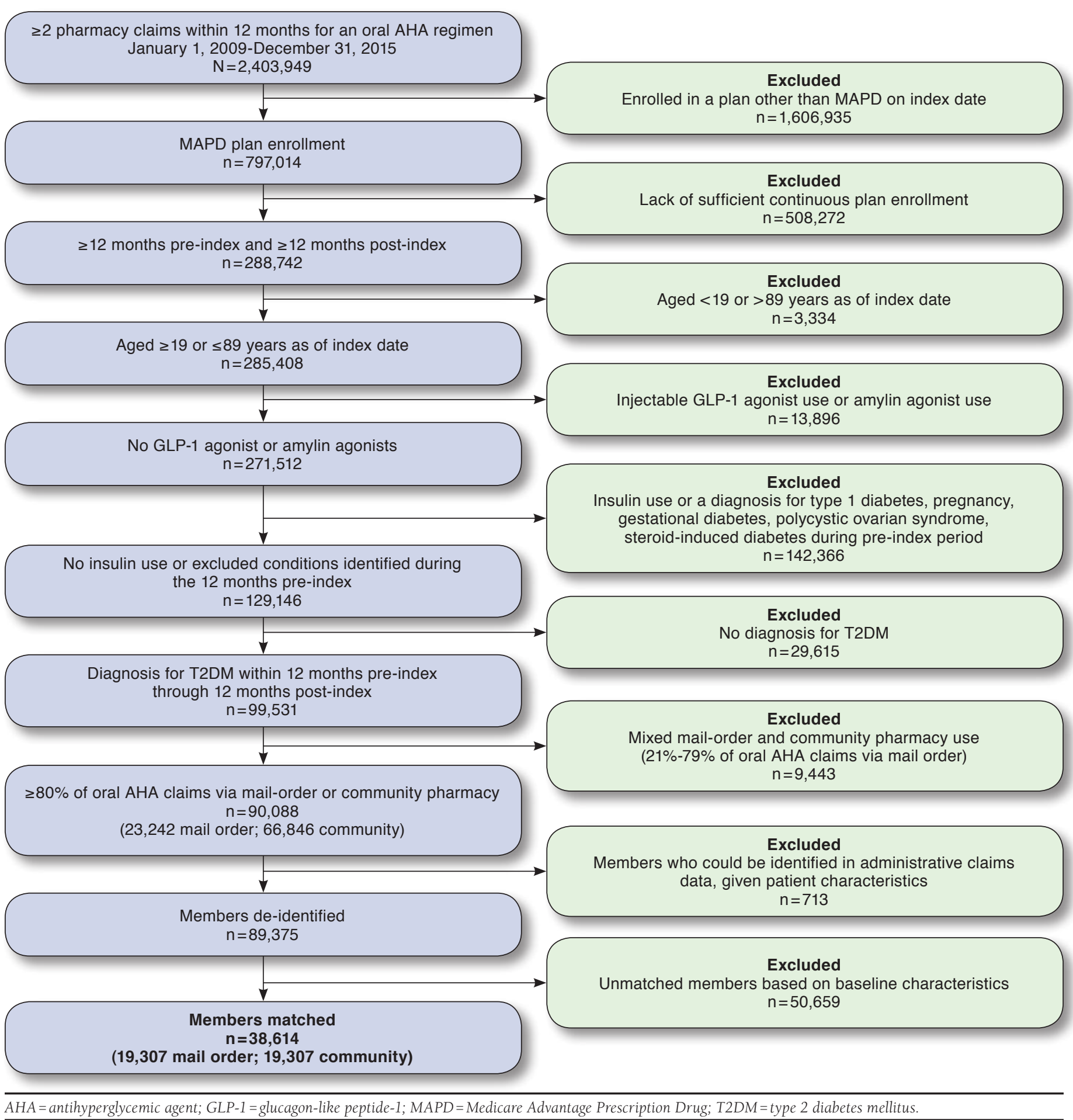

AHA = antihyperglycemic agent; GLP-1 = glucagon-like peptide-1; MAPD = Medicare Advantage Prescription Drug; T2DM = type 2 diabetes mellitus. 


\section{A Retrospective Database Study Comparing Diabetes-Related Medication Adherence and Health Outcomes for Mail-Order Versus Community Pharmacy}

\begin{tabular}{|c|c|c|c|c|c|c|c|c|}
\hline \multirow[b]{2}{*}{ Time Period } & \multicolumn{2}{|c|}{12 Months } & \multicolumn{2}{|c|}{24 Months } & \multicolumn{2}{|c|}{36 Months } & \multicolumn{2}{|c|}{48 Months } \\
\hline & Mail Order & Community & Mail Order & Community & Mail Order & Community & Mail Order & Community \\
\hline $\mathrm{n}$ & 19,307 & 19,307 & 9,437 & 9,437 & 4,455 & 4,455 & 2,032 & 2,032 \\
\hline PDC,, mean $( \pm S D)$ & $0.93( \pm 0.14)$ & $0.82( \pm 0.24)$ & $0.89( \pm 0.18)$ & $0.79( \pm 0.26)$ & $0.88( \pm 0.20)$ & $0.78( \pm 0.27)$ & $0.87( \pm 0.21)$ & $0.77( \pm 0.28)$ \\
\hline Patient adherence, a \% & 85.9 & 68.1 & 81.1 & 64.6 & 79.6 & 63.1 & 78.4 & 62.4 \\
\hline
\end{tabular}

36-, and 48-month PDC separately. The proportion of patients adherent to oral AHAs was compared using McNemar's test based on the same time periods.

For Alc analyses, only those patients with an Alc result in the respective period were included for the analysis of that period. For patients with an insulin claim or other injectable claim in the post-index period, only those Alc measures occurring before the first insulin or other injectable claim were evaluated. Paired t-tests were used to compare Alc change from baseline to 12 months, baseline to 24 months, baseline to 36 months, and baseline to 48 months, separately. Alc goal achievement was compared using McNemar's test based on patients with 12-, 24-, 36-, and 48-month Alc results, separately.

ED visits and inpatient hospitalizations were treated as binary variables based on evidence of an event within the respective 12-, 24-, 36-, or 48-month periods and were compared using McNemar's test, separately for each period.

\section{Results}

\section{Study Sample}

Matched cohorts of 19,307 patients who predominantly used mail-order pharmacies and 19,307 patients who predominantly used community pharmacies were included for the study analyses (Figure 1). The study data show that 90-day supplies were more widely used in mail-order pharmacies, as expected, with approximately $90 \%$ of mail-order claims being 90 -days of supply, while $<10 \%$ of community pharmacy claims were for 90-days of supply. Subsequently, PDC was higher for mail-order pharmacy patients at 12 months $(0.93 \pm 0.14$ vs. $0.82 \pm 0.24, P<0.001)$, at 24 months $(0.89 \pm 0.18$ vs. $0.79 \pm 0.26$, $P<0.001)$, at 36 months $(0.88 \pm 0.20$ vs. $0.78 \pm 0.27, P<0.001)$, and at 48 months $(0.87 \pm 0.21$ vs. $0.77 \pm 0.28, P<0.001$; Table 1$)$. Corresponding to higher PDC, adherence (proportion with $P D C \geq 0.8$ ) was also greater for mail-order pharmacy patients through 12 months ( $86 \%$ vs. $68 \%, P<0.001), 24$ months ( $81 \%$ vs. $65 \%, P<0.001), 36$ months ( $80 \%$ vs. $63 \%, P<0.001)$, and 48 months (78\% vs. $62 \%, P<0.001$; Table 1 ).
Although both groups (mail-order and community pharmacy) had relatively high PDC, the mail-order pharmacy group also demonstrated slightly better outcomes in terms of glycemic control, despite relatively small numbers of patients with sufficient Alc results within the Humana Research Database ( $n=3,193$ per matched cohort at 12 months, $n=1,389$ at 24 months, $\mathrm{n}=589$ at 36 months, $\mathrm{n}=370$ at 48 months; Table 2). Mail-order pharmacy patients had marginally, not statistically significant, greater reductions in Alc level. Glycemic control for the mail-order pharmacy group was observed at 12 months (91\% vs. $89 \%, P=0.006$ ), 24 months (91\% vs. $88 \%, P=0.009$ ), and 36 months $93 \%$ vs. $89 \%, P=0.043$ ), although not at 48 months ( $89 \%$ vs. $89 \%, P=0.10$ ) when evaluating goal achievement using the less-stringent measure of $\mathrm{Alc}<8 \%$. The effect was not detectable when Alc goal achievement was measured as $\mathrm{Alc}<7 \%$.

Despite similar Alc levels for mail-order and community pharmacy groups at baseline (7.44 \pm 1.3 and $7.43 \pm 1.3$; Table 3), post hoc evaluation of baseline goal achievement revealed marginally greater, but not statistically significant, achievement at Alc $<8 \%$ for the mail-order pharmacy group (76\% vs. $74 \%, P=0.052$ ) and marginally lower, but not statistically significant, achievement at Alc $<7 \%$ for the mail-order pharmacy group ( $40 \%$ vs. $41 \%, P=0.476$ ) for those patients who also had Alc results at 12 months. Baseline goal achievement proportions were similarly distributed for patients with Alc results at 24 months $(79 \%$ vs. $77 \%$ Alc $<8 \%, P=0.253 ; 43 \%$ vs. $43 \%$ Alc $<7 \%, P=0.818)$ where baseline Alc was $7.33( \pm 1.18)$ versus $7.37( \pm 1.21)$ for the mail-order versus community pharmacy group. Baseline data for patients with Alc results at 36 months were also similarly categorized ( $82 \%$ vs. $80 \%$, Alc $<8 \%$, $P=0.297 ; 47 \%$ vs. $47 \%$, Alc $<7 \%, P=1.0)$ where baseline Alc was $7.22( \pm 1.08)$ versus $7.28( \pm 1.16)$ for the mail-order versus community pharmacy group. In summary, marginal differences in proportion of patients meeting Alc $<8 \%$ at baseline were not statistically significant, and marginal differences observed at 12 months, 24 months, and 36 months were statistically significant.

In addition to slightly more favorable glycemic control, as measured by Alc, the mail-order pharmacy cohort had 


\section{A Retrospective Database Study Comparing Diabetes-Related Medication Adherence and Health Outcomes for Mail-Order Versus Community Pharmacy}

\begin{tabular}{|c|c|c|c|c|c|c|c|c|}
\hline \multirow[b]{2}{*}{ Time Point } & \multicolumn{2}{|c|}{12 Months } & \multicolumn{2}{|c|}{24 Months } & \multicolumn{2}{|c|}{36 Months } & \multicolumn{2}{|c|}{48 Months } \\
\hline & Mail Order & Community & Mail Order & Community & Mail Order & Community & Mail Order & Community \\
\hline $\mathrm{n}$ & 3,193 & 3,193 & 1,389 & 1,389 & 589 & 589 & 370 & 370 \\
\hline$\%$ Alc, difference $( \pm$ SE) & $-0.60( \pm 0.02)$ & $-0.58( \pm 0.02)$ & $-0.52( \pm 0.03)$ & $-0.50( \pm 0.03)$ & $-0.45( \pm 0.05)$ & $-0.44( \pm 0.05)$ & $-0.33( \pm 0.06)$ & $-0.47( \pm 0.06)$ \\
\hline Alc $<8 \%$ (\% meeting goal) & $91.1^{\mathrm{a}}$ & $89.0^{\mathrm{a}}$ & $90.9^{a}$ & $87.8^{\mathrm{a}}$ & $92.5^{b}$ & $89.1^{\mathrm{b}}$ & 88.6 & 88.6 \\
\hline Alc $<7 \%$ (\% meeting goal) & 65.8 & 65.0 & 67.0 & 64.4 & 68.9 & 64.0 & 67.3 & 68.1 \\
\hline \multicolumn{9}{|c|}{$\begin{array}{l}\text { Differences between mail-order and community cohorts were evaluated for each time period using a paired t-test for Alc change and McNemar's test for proportion } \\
\text { achieving Alc goal. }\end{array}$} \\
\hline
\end{tabular}

fewer patients with an ED visit within 12 months (26\% vs. $28 \%, P<0.0001$ ), within 24 months ( $41 \%$ vs. $44 \%, P<0.001$ ), and within 36 months (50\% vs. 54\%, $P<0.0001$; Table 4). Proportional differences within 48 months were not statistically significant (58\% vs. $60 \%, P=0.168)$. Similarly, fewer patients in the mail-order pharmacy cohort had an inpatient hospitalization within 12 months (17\% vs. 19\%, $P<0.0001$ ), within 24 months ( $28 \%$ vs. $31 \%, P<0.001$ ), within 36 months (36\% vs. $40 \%, P<0.0001)$, and within 48 months ( $43 \%$ vs. $47 \%, P=0.009$ ).

\section{Discussion}

Consistent with previous studies, this study found that mailorder pharmacy users were more adherent to oral AHAs than community pharmacy users. The $86 \%$ of mail-order pharmacy users and $68 \%$ of community pharmacy users who were adherent after 12 months in this study is most similar to research conducted by Divine et al. (2010), which found 82\% of mail-order pharmacy users and 63\% of community pharmacy users adherent. ${ }^{9}$ Although both studies used propensity score matching to focus on the effect of pharmacy distribution channel (mail order vs. community), the work conducted by Divine et al. (2010) analyzed MarketScan data, which largely comprised patients enrolled in commercial, employer-sponsored health plans. ${ }^{8}$ In contrast, our study used MAPD claims data from the Humana Research Database, which highlights the consistency of findings across the populations. The relationship between mail-order pharmacy use and greater adherence to oral AHAs has been observed across multiple administrative claims data sources, representing a significant portion of the U.S. population. ${ }^{7-12}$

There may be concerns that the evidence in favor of mailorder pharmacy is simply an artifact of claims data where mailorder pharmacy use of 90 days of supply fills with refills creates the appearance of greater adherence without evidence of actual medication consumption. However, the present study focused on patients enrolled in MAPD plans where patients must initiate or authorize each mail-order refill, and this study found a clear and sustained greater adherence for mail-order pharmacy users over 24,36 , and 48 months, suggesting the positive adherence effect is not simply an artifact of mail-order pharmacy claims data. Previous studies comparing mail-order and community pharmacies have limited their observations of adherence and other outcomes to approximately 12 months and may have been more susceptible to bias from 90-day supplies.

The results of this study also demonstrate a positive relationship between adherence and glycemic control when measured as Alc $<8 \%$. The effect on glycemic control was observed within 12 months and was sustained over 24 and 36 months. The effect was not observed through 48 months, and the nominal differences were actually in conflict with earlier periods, but the sample had decreased substantially from those with a 12-month Alc result. Similarly, the effect on glycemic control, measured as Alc $<7 \%$, and differences in Alc level were numerically greater for mail-order pharmacy users at 12, 24, and 36 months, yet lower at 48 months, but the differences were not statistically significant. Although the downstream effect of greater adherence may not be strong enough to result in measurable differences in the decrease of Alc level or in differences in the proportions of patients achieving Alc $<7 \%$, the nonsignificant results in the study were possibly a function of insufficient power to detect small differences. Differences in proportions of patients achieving Alc $<8 \%$ were significant and sustainable, in agreement with the theoretical association between adherence and glycemic control.

Post hoc analysis of baseline Alc goal achievement suggests that the effect on Alc in this study was very small, but likely meaningful. Despite having similar average levels of Alc at baseline, the mail-order pharmacy group actually included a marginally greater proportion of patients with Alc $<8 \%$ but a marginally smaller proportion of patients with $\mathrm{Alc}<7 \%$. These marginal differences were not statistically significant. In the outcomes measurement periods of 12,24 , and 36 months, the greater proportional difference in Alc $<8 \%$ for the mail-order pharmacy group was statistically significant. Additionally, the 


\section{A Retrospective Database Study Comparing Diabetes-Related Medication Adherence and Health Outcomes for Mail-Order Versus Community Pharmacy}

\begin{tabular}{|c|c|c|c|c|c|c|c|c|c|c|c|c|c|}
\hline & \multicolumn{3}{|c|}{ Before Matching } & \multicolumn{3}{|c|}{ After Matching } & & \multicolumn{3}{|c|}{ Before Matching } & \multicolumn{3}{|c|}{ After Matching } \\
\hline & $\begin{array}{l}\text { Mail } \\
\text { Order }\end{array}$ & Community & $\begin{array}{l}\text { Std } \\
\text { Diff }\end{array}$ & $\begin{array}{c}\text { Mail } \\
\text { Order }\end{array}$ & Community & $\begin{array}{l}\text { Std } \\
\text { Diff }\end{array}$ & & $\begin{array}{c}\text { Mail } \\
\text { Order }\end{array}$ & Community & \begin{tabular}{|l|} 
Std \\
Diff
\end{tabular} & $\begin{array}{l}\text { Mail } \\
\text { Order }\end{array}$ & Community & $\begin{array}{l}\text { Std } \\
\text { Diff }\end{array}$ \\
\hline Sample size, $\mathrm{n}$ & 23,120 & 66,255 & & 19,307 & 19,307 & & \multicolumn{7}{|c|}{ Humana segmentation, \% } \\
\hline \multicolumn{7}{|l|}{ Age, years, $\%$} & Chronic condition & segment & & & & & \\
\hline $31-50$ & 2.7 & 1.2 & -10.5 & 1.4 & 1.4 & -0.4 & Chronic 6 & 5.5 & 0.2 & -32.4 & 0.2 & 0.2 & 0.7 \\
\hline $51-60$ & 8.4 & 7.0 & -5.2 & 7.6 & 7.5 & -0.1 & Chronic 7 & 1.4 & 0.7 & -6.6 & 0.8 & 0.9 & 0.8 \\
\hline $61-70$ & 38.2 & 42.6 & 8.9 & 42.4 & 42.3 & -0.3 & \multicolumn{7}{|c|}{ Health behaviors segments } \\
\hline $71-80$ & 39.2 & 40.9 & 3.4 & 39.9 & 40.1 & 0.5 & Behaviors 1 & 10.8 & 32.0 & 53.5 & 29.5 & 29.4 & -0.3 \\
\hline $81-89$ & 11.5 & 8.3 & -10.6 & 8.7 & 8.7 & 0.0 & Behaviors 2 & 3.9 & 11.6 & 29.0 & 10.6 & 10.6 & 0.0 \\
\hline \multicolumn{7}{|l|}{ Gender, \% } & Behaviors 3 & 3.5 & 9.6 & 24.6 & 9.0 & 9.0 & 0.1 \\
\hline Female & 50.5 & 46.9 & -7.4 & 48.4 & 48.4 & -0.2 & Behaviors 4 & 0.4 & 0.4 & -0.8 & 0.5 & 0.4 & -0.8 \\
\hline Male & 49.5 & 53.1 & 7.4 & 51.6 & 51.6 & 0.2 & Behaviors 5 & 11.1 & 0.4 & -47.2 & 0.4 & 0.5 & 0.3 \\
\hline \multicolumn{7}{|l|}{ Race, \% } & Behaviors 6 & 9.4 & 0.3 & -43.3 & 0.4 & 0.3 & -0.8 \\
\hline White & 75.6 & 85.1 & 24.3 & 83.1 & 83.3 & 0.6 & Behaviors 7 & 7.4 & 0.3 & -37.8 & 0.3 & 0.3 & 0.2 \\
\hline Black & 16.5 & 10.3 & -18.2 & 11.9 & 11.6 & -1.0 & Behaviors 8 & 1.4 & 0.1 & -14.9 & 0.1 & 0.1 & 0.6 \\
\hline Other & 7.9 & 4.5 & -14.2 & 5.0 & 5.1 & 0.5 & Not assigned to & 4.8 & 3.8 & -5.0 & 4.3 & 4.5 & 0.9 \\
\hline \multicolumn{7}{|c|}{ Population density, $\%$} & a segment & \multirow{3}{*}{49.7} & \multirow{3}{*}{50.4} & \multirow{3}{*}{1.3} & \multirow{3}{*}{51.2} & \multirow{3}{*}{50.8} & \\
\hline Urban & 66.5 & 55.7 & -22.3 & 58.3 & 58.1 & -0.3 & \multirow{2}{*}{$\begin{array}{l}\text { Pre-index use of } \\
\text { oral AHA, \% }\end{array}$} & & & & & & \multirow[t]{2}{*}{-0.6} \\
\hline Suburban & 22.8 & 29.8 & 15.9 & 28.5 & 28.4 & -0.2 & & & & & & & \\
\hline Rural & 10.0 & 13.7 & 11.3 & 12.4 & 12.7 & 0.8 & \multirow{4}{*}{$\begin{array}{l}\text { Rx-Risk score, } \\
\text { mean }( \pm S D) \\
\text { DCSI, mean }( \pm S D)\end{array}$} & \multirow{2}{*}{$\begin{array}{c}5.72 \\
( \pm 3.0)\end{array}$} & \multirow{2}{*}{$\begin{array}{c}5.60 \\
( \pm 2.7)\end{array}$} & \multirow[t]{2}{*}{-4.1} & \multirow{2}{*}{$\begin{array}{c}5.69 \\
( \pm 2.9)\end{array}$} & \multirow{2}{*}{$\begin{array}{c}5.68 \\
( \pm 2.7)\end{array}$} & \multirow[t]{2}{*}{-0.3} \\
\hline Unknown & 0.7 & 0.8 & 1.9 & 0.8 & 0.8 & -0.2 & & & & & & & \\
\hline Region, \% & & & & & & & & 2.00 & 1.66 & -18.2 & 2.65 & 2.65 & 0.1 \\
\hline Northeast & 1.9 & 2.7 & 5.0 & 2.4 & 2.4 & -0.3 & & & & & $( \pm 1.6)$ & & \\
\hline Midwest & 17.1 & 27.9 & 26.2 & 24.3 & 24.5 & 0.6 & $\begin{array}{l}\text { Baseline Alc level, } \\
\text { mean }( \pm S D)\end{array}$ & $\begin{array}{c}7.52 \\
( \pm 1.4)\end{array}$ & $\begin{array}{c}7.43 \\
( \pm 1.3)\end{array}$ & -17.9 & $\begin{array}{l}7.44 \\
( \pm 1.3)\end{array}$ & $\begin{array}{l}7.43 \\
( \pm 1.3)\end{array}$ & -0.1 \\
\hline South & $\frac{73.2}{578}$ & $\begin{array}{l}57.9 \\
11.5\end{array}$ & $\frac{-32.7}{128}$ & $\begin{array}{ll}63.1 \\
102\end{array}$ & $\frac{62.4}{107}$ & \begin{tabular}{|r|}
-1.4 \\
14
\end{tabular} & Baseline ED use, \% & 31.7 & 24.5 & -16.0 & 27.0 & 27.0 & -0.2 \\
\hline$\frac{\text { West }}{\text { Low income statu }}$ & 57.8 & 11.5 & 12.8 & 10.2 & 10.7 & 1.4 & Baseline & 21.1 & 16.4 & -11.9 & 18.3 & 18.0 & -0.7 \\
\hline LIS only & 7.8 & 5.1 & -11.0 & 5.8 & 5.8 & 0.0 & hospitalization, \% & & & & & & \\
\hline Medicaid only & 0.5 & 0.5 & 0.5 & 0.5 & 0.5 & 0.1 & $\begin{array}{l}\text { Baseline outpatient } \\
\text { use, mean }( \pm S D)\end{array}$ & $\begin{array}{l}16.9 \\
( \pm 16)\end{array}$ & $\begin{array}{l}16.3 \\
( \pm 14)\end{array}$ & -4.1 & $\begin{array}{l}16.9 \\
( \pm 15)\end{array}$ & $\begin{array}{l}16.8 \\
( \pm 14)\end{array}$ & -0.6 \\
\hline $\begin{array}{l}\text { LIS and Medicaid } \\
\text { eligible }\end{array}$ & 23.7 & 10.4 & -36.0 & 12.1 & 12.2 & 0.2 & Baseline pharmacy & 6.83 & 6.81 & -1.7 & 6.86 & 6.85 & -0.5 \\
\hline No status & 68.0 & 84.0 & 38.1 & 81.5 & 81.5 & -0.2 & $\begin{array}{l}\text { costs }(\log ), \text { mean } \\
( \pm \text { SD })\end{array}$ & & & & & $( \pm 1.5)$ & \\
\hline Humana segmentat & on, $\%$ & & & & & & Baseline medical & 8.06 & 8.03 & -2.6 & 8.08 & 8.07 & -0.3 \\
\hline Chronic conditior & segmen & & & & & & costs $(\log )$, mean & $( \pm 1.4)$ & $( \pm 1.3)$ & & $( \pm 1.3)$ & $( \pm 1.3)$ & \\
\hline Chronic 1 & 2.9 & 4.0 & 5.7 & 4.6 & 4.5 & -0.6 & $( \pm$ SD $)$ & & & & & & \\
\hline Chronic 2 & 14.9 & 20.7 & 15.3 & 23.6 & 23.5 & -0.4 & Baseline total costs & 8.54 & 8.48 & -5.6 & 8.54 & 8.53 & -0.6 \\
\hline Chronic 3 & 2.7 & 8.9 & 26.8 & 7.4 & 7.6 & 0.8 & $(\log )$, mean $( \pm S D)$ & $( \pm 1.2)$ & $( \pm 1.1)$ & & $( \pm 1.1)$ & $( \pm 1.1)$ & \\
\hline Chronic 4 & 4.8 & 6.6 & 8.0 & 7.6 & 7.6 & 0.0 & Presence of & 17.4 & 13.0 & -12.5 & 14.7 & 14.3 & -1.1 \\
\hline Chronic 5 & 15.0 & 0.4 & -57.0 & 0.6 & 0.5 & -1.1 & post-index Alc, \% & & & & & & \\
\hline
\end{tabular}

Note: Costs were log-transformed.

Alc=hemoglobin Alc; AHA = antihyperglycemic agent; DCSI = Diabetes Complications Severity Index; ED =emergency department; LIS=low-income subsidy status; $S D=$ standard deviation; Std Diff = standardized differences.

proportions achieving Alc $<7 \%$ were nominally greater in the outcomes periods of 12,24 , and 36 months, even though they were nominally lower in the baseline period for the 12- and 24-month analyses and equal for the 36-month analysis.

If small sample sizes were a factor in the lack of statistically significant findings for the Alc $<7 \%$ analysis, a future study would need to include data for a much greater number of patients. Detection of a difference between $68.9 \%$ and $64.0 \%$, as seen in the 36-month analysis, would require about 1,400 patients or more per group, while detection of $65.8 \%$ versus $65.0 \%$, as seen in the 12 -month analysis, might require as many as 55,000 patients or more per group. Similarly, detection of the marginal -0.02 difference in Alc level decrease observed at 12 months and at 24 months would have required 20,000 or more patients per group for $80 \%$ power.

While the effect on Alc was very small, the direction of effect was toward the mail-order pharmacy group for the most part in this study. Small effects can be meaningful at the 


\section{A Retrospective Database Study Comparing Diabetes-Related Medication Adherence and Health Outcomes for Mail-Order Versus Community Pharmacy}

\begin{tabular}{l} 
TABLE 4 Comparisons of Mail-Order and Community Pharmacy Users on ED Visits and \\
Inpatient Hospitalizations \\
\hline
\end{tabular}

population level, especially when applied to long-term medication use and clinical outcomes and due to the potential effect on performance or quality measures for health plans and providers who participate in value-based payment schemes.

The present study demonstrates that mail-order pharmacy users were less likely to have an ED visit and less likely to have an inpatient hospitalization. The effect on ED visits was statistically significant within 12 months and was sustained through 36 months, while the effect on inpatient hospitalization was sustained through 48 months. The finding follows the theoretical connection between greater adherence and better health outcomes for mail-order pharmacy users. The evidential connection is further strengthened by the Alc data because mail-order pharmacy users were more likely to achieve glycemic control (Alc $<8 \%)$.

The preferential outcomes for the mail-order pharmacy cohort, combined with the stability of greater adherence for the mail-order pharmacy cohort supports the notion that patients receiving oral AHAs via mail order actually consumed more medication than patients receiving medications via community pharmacy, despite concerns that pharmacy claims data do not confirm actual use of medications.

Another concern with mail-order and community pharmacy comparisons arises from potential self-selection to mailorder pharmacy by patients who are inherently more likely to be adherent. Matching methods can be applied to mitigate such differences between mail-order pharmacy users and community pharmacy users, but matching can only consider measured variables. Although it is possible that there were important, unmeasured characteristic differences between the cohorts in the present study, the study accounted for a fairly comprehensive range of variables in order to minimize the possibility that unmeasured differences between the cohorts influenced the outcomes.

\section{Limitations}

Limitations related to the data source should be considered when interpreting the findings. The study results may be more generalizable to patients in the South and Midwest as opposed to the Northeast and West regions of the United States, given the location of the individuals in the study population. Additionally, availability of Alc results in the Humana Research Database may be more likely for patients residing in the South due to market coverage of laboratory suppliers that provide data to Humana; however, T2DM is more prevalent in the South than in other regions in the general population. ${ }^{29}$

\section{Conclusions}

Mail-order pharmacy provides patients with the convenience of home delivery along with greater use of 90 days of supply as a normal practice, both of which should facilitate availability of medication supply for patients taking chronic medications beyond what patients can expect through community pharmacies. The present study confirmed a benefit of using mail-order pharmacy within an MAPD population of patients using chronic, oral AHAs to treat T2DM in terms of medication adherence along with better glycemic control and preferential health care use outcomes. These benefits were observed in the near term and were sustained over time.

\section{Authors}

PHIL SCHWAB, PhD; PATRICK RACSA, MS; and KAREN WORLEY, PhD, Humana Healthcare Research, Louisville, Kentucky. KAREN RASCATI, RPh, PhD, The University of Texas at Austin. MARC MOURER, MBA, and YUNUS MEAH, PharmD, Humana Pharmacy Solutions, Louisville, Kentucky.

AUTHOR CORRESPONDENCE: Phil Schwab, PhD, Humana Healthcare Research, 515 W. Market, Louisville, KY 40202.

Tel.: 502.476.5610; E-mail: pschwab@humana.com. 


\section{A Retrospective Database Study Comparing Diabetes-Related Medication Adherence and Health Outcomes for Mail-Order Versus Community Pharmacy}

\section{DISCLOSURES}

This study received no outside funding but was sponsored by Humana through regular employment activities by Schwab, Racsa, and Worley, who are employed by Humana Healthcare Research (formerly Comprehensive Health Insights). This study found benefits related to using mail-order versus community pharmacies for dispensing antihyperglycemic agents in the treatment of type 2 diabetes. Humana owns mail-order pharmacies under the Humana Pharmacy subsidiary. Mourer and Meah are paid employees of Humana Pharmacy Solutions. Rascati is employed by the University of Texas College of Pharmacy at Austin.

\section{REFERENCES}

1. Lau DT, Nau DP. Oral antihyperglycemic medication nonadherence and subsequent hospitalization among individuals with type 2 diabetes. Diabetes Care. 2004:27(9):2149-53

2. Sokol MC, McGuigan KA, Verbrugge RR, Epstein RS. Impact of medication adherence on hospitalization risk and healthcare cost. Med Care. 2005;43(6):521-30

3. Lawrence DB, Ragucci KR, Long LB, Parris BS, Helfer LA. Relationship of oral antihyperglycemic (sulfonylurea or metformin) medication adherence and hemoglobin Alc goal attainment for HMO patients enrolled in a diabetes disease management program. J Manag Care Pharm. 2006;12(6):466-71. Available at: https://www.jmcp.org/doi/10.18553/jmcp.2006.12.6.466.

4. Taitel M, Fensterheim L, Kirkham H, Sekula R, Duncan I. Medication days' supply, adherence, wastage, and cost among chronic patients in Medicaid. Medicare Medicaid Res Rev. 2012;2(3):pii:mmrr.002.03.a04.

5. Schmittdiel JA, Karter AJ, Dyer W, Chan J, Duru OK. The safety and effectiveness of mail order pharmacy use in diabetes patients. Am J Manag Care. 2013;19(11):882-87.

6. Fernandez EV, McDaniel JA, Carroll NV. Examination of the link between medication adherence and use of mail-order pharmacies in chronic disease states. J Managed Care Spec Pharm. 2016;22(11):1247-59. Available at: https:// www.jmcp.org/doi/10.18553/jmcp.2016.22.11.1247.

7. Duru OK, Schmittdiel JA, Dyer WT, et al. Mail-order pharmacy use and adherence to diabetes-related medications. Am J Manag Care. 2010;16(1):33-40.

8. Devine S, Vlahiotis A, Sundar H. A comparison of diabetes medication adherence and healthcare costs in patients using mail order pharmacy and retail pharmacy. J Med Econ. 2010;13(2):203-11.

9. Zhang L, Zakharyan A, Stockl KM, et al. Mail-order pharmacy use and medication adherence among Medicare Part D beneficiaries with diabetes. J Med Econ. 2011;14(5):562-67.

10. Khandelwal N, Duncan I, Rubinstein E, et al. Medication adherence for 90-day quantities of medication dispensed through retail and mail order pharmacies. Am J Manag Care. 2011;17(11):e427-34.

11. Sharma KP, Taylor TN. Pharmacy effect on adherence to antidiabetic medications. Med Care. 2012;50(8):685-91.

12. Kirkman MS, Rowan-Martin MT, Levin R, et al. Determinants of adherence to diabetes medications: findings from a large pharmacy claims database. Diabetes Care. 2015;38(4):604-09.
13. Sloan KL, Sales AE, Liu CF, et al. Construction and characteristics of the RxRisk-V: a VA-adapted pharmacy-based case-mix instrument. Med Care. 2003;4l(6):761-64.

14. Young BA, Lin E, Von Korff M, et al. Diabetes complications severity index and risk of mortality, hospitalization, and healthcare utilization. Am J Manag Care. 2008;14(1):15-23.

15. Chang H-Y, Weiner JP, Richards TM, et al. Validating the adapted Diabetes Complications Severity Index in claims data. Am J Manag Care. 2012;18(11):721-26

16. Hart LG, Larson EH, Lishner DM Rural definitions for health policy and research. Am J Public Health. 2005;95(7):1149-55.

17. West AN, Weeks WB, Wallace AE. Rural veterans and access to highquality care for high-risk surgeries. Health Serv Res. 2008;43(5 Pt 1):1737-51. 18. Klabunde Potosky AL, Legler JM, Warren JL. Development of a comorbidity index using physician claims data. J Clin Epidemiol. 2000;53(12):1258-67.

19. Sales AE, Liu CF, Sloan KL, et al. Predicting costs of care using a pharmacy-based measure risk adjustment in a veteran population. Med Care. 2003;4l(6):753-60.

20. Fishman PA, Goodman MJ, Hornbrook MC, Meenan RT, Bachman DJ, O'Keeffe Rosetti MC. Risk adjustment using automated ambulatory pharmacy data: the RxRisk model. Med Care. 2003;41(1):84-99.

21. Farley JF, Harley CR, Devine JW. A comparison of comorbidity measurements to predict healthcare expenditures. Am J Manag Care. 2006;12(2):110-19.

22. Johnson ML, El-Serag HB, Tran TT, Hartman C, Richardson P, Abraham NS. Adapting the Rx-Risk-V for mortality prediction in outpatient populations. Med Care. 2006;44(8):793-97.

23. Fan VS, Maciejewski ML, Liu C-F, McDonell MB, Fihn SD. Comparison of risk adjustment measures based on self-report, administrative data, and pharmacy records to predict clinical outcomes. Health Serv Outcomes Res Methodol. 2006;6(1):21-36.

24. Vitry A, Wong SA, Roughead EE, Ramsay E, Barratt J. Validity of medication-based co-morbidity indices in the Australian elderly population. Aust N Z J Public Health. 2009;33(2):126-30.

25. Lu CY, Barratt J, Vitry A, Roughead E. Charlson and Rx-Risk comorbidity indices were predictive of mortality in the Australian health care setting. J Clin Epidemiol. 2011;64(2):223-28.

26. American Diabetes Association. Glycemic targets: standards of medical care in diabetes-2018. Diabetes Care. 2018;41(Suppl 1):S55-S64.

27. Qaseem A, Wilt TJ, Kansagara D, et al. Hemoglobin Alc targets for glycemic control with pharmacologic therapy for nonpregnant adults with type 2 diabetes mellitus: a guidance statement update from the American College of Physicians. Ann Intern Med. 2018;168(8):569-76.

28. National Committee for Quality Assurance (NCQA). Comprehensive diabetes care. Available at: https://www.ncqa.org/hedis/measures/comprehensive-diabetes-care/. Accessed January 10, 2019.

29. Frost A, Hiers S, Herrera C-N. The prevalence of diagnosed diabetes, pre-diabetes, and gestational diabetes among the ESI population, 2008-2012. Health Care Cost Institute. Issue brief no. 6. December 2013 Available at: https://www.healthcostinstitute.org/images/easyblog_articles/117/Diabetes-Prevalence-2008-2012.pdf. Accessed January 10, 2019. 